\title{
Uniqueness of Tchebycheff Spaces and their Ideal Relatives
}

\author{
Boris Shekhtman \\ Dept. of Mathematics \\ University of South Florida \\ Tampa, Fl. 33620, USA. \\ boris@math.usf.edu \\ To the memory of my friend and teacher Ambikeshwar Sharma
}

June 24, 2005

\begin{abstract}
In the first part of the paper we show that the space of polynomials of degree $n-1$ is the unique $n$-dimensional Tchebycheff subspace of polynomials. We also define a generalization of Tchebycheff spaces: "Ideal complements" and demonstrate their uniqueness.

In the second part we discuss various analogues of Tchebycheff spaces (minimal interpolating systems) in several variables.
\end{abstract}

\section{Preface:}

I first met Professor Sharma twenty seven years ago. I was a young graduate student, my head was filled with "Bourbakisms", my Ph.D. thesis was about interpolation (in Banach spaces, of course) and I was looking forward to learn more from the renown expert in the field. To my surprise Sharma told me that he didn't understand what a functional was and the only theorems worth knowing in analysis was the Taylor formula and maybe "integration by parts" although he had his doubts about the latter. With typical modesty, he told me that he wasn't bright enough for the abstractions. The best he could do, was to compute a few "right" examples and hope to get lucky. That send me for a spin, that lasted awhile. I tried to "compute" with Sharma only to learn that there is no way for me to keep up with his speed and accuracy. I believe that this was a lesson learned by many of my colleagues. Fortunately "Maple" came about and like "Colt 45", equalized the playing field.

This paper is about solvability of various interpolation problems and its generalizations, the topic that benefited greatly by many contributions of Sharma and his collaborators (cf. [4-7], [10], [12], [16-19]).

The paper is divided into two parts. The aim of the first part is to investigate the general form and uniqueness of Tchebycheff and Extended Tchebycheff subspaces as well as "ideal complements" in the spaces of polynomials. In particular 
we will show that the space of complex polynomials of degree at most $n-1$ is unique Tchebycheff subspace of polynomials. We also introduce a new definition of "an ideal complement" which is formally stronger then that of a Tchebycheff and Extended Tchebycheff subspace and study the form and uniqueness of ideal complements.

In the second part we discuss various generalizations of Tchebycheff subspaces and ideal complements in several variables. In section 2.1 we introduce "minimal $k$-interpolating spaces" as a generalization of the notion of Tchebycheff spaces and "minimal $k$-ideal complements". We investigate the dimension of these spaces. In section 2.2 we introduce another possible generalization of Tchebycheff subspaces and ideal complements in several variables: namely "minimal family of $k$-interpolating spaces" and "minimal family of $k$-ideal complements". While the investigation in section 2.1 has a distinct topological nature, the methods used in this section 2.2 are mostly of combinatorial type. Unlike the Tchebycheff spaces, their analogs in several variables have not received much attention in the literature. Therefore it is not surprising, that the ratio of the number of theorems to open problems in this part is rather small.

Going back to my early years, I was convinced that the only obstacle in generalizing results in one variable is unbearable notations in several. By now I know better. Yet the original hindrance remains. Given survey stile of this article, I will take a "poetic license" not to dwell on self-evident notations, hence saving trees and not trying the patience of a reader. For the same reason, I will customarily give the simple proofs of a theorem and refer to an original article for more complicated ones. As the wise man said: "a simple example explains the situation much better".

\section{Interpolating Spaces in One Variable}

All that being said, here are some notations:

Let $\mathbb{F}$ either be the field of real or that of the complex numbers and $\mathbb{F}[x]$ be the ring of polynomials with coefficients in $\mathbb{F}$. As such, $\mathbb{F}[x]$ is a linear space over the field $\mathbb{F}$. We use $\mathbb{F}_{<n}[x]$ to denote the space of polynomials of degree less than $n$; i.e.

$$
\mathbb{F}_{<n}[x]:=\operatorname{span}\left[1, x, \ldots, x^{n-1}\right] \subset \mathbb{F}[x] .
$$

An $n$-dimensional subspace $V \subset \mathbb{F}[x]$ is called Tchebycheff (cf. [13], [14]) if

$$
f \in V \text { and } f\left(x_{j}\right)=0 ; j=1, \ldots, n
$$

for a distinct set of points $\Delta:=\left\{x_{1}, x_{2}, \ldots, x_{n}\right\} \subset \mathbb{F}$ implies $f=0$. That is every non-zero $f \in V$ has at most $n$ distinct zeroes.

Equivalently (cf. [13], [14]) an $n$-dimensional space $V \subset \mathbb{F}[x]$ is Tchebycheff if and only if it is interpolating:

For any distinct set of points $\Delta:=\left\{x_{1}, x_{2}, \ldots, x_{n}\right\} \subset \mathbb{F}$ and any set of values $a_{1}, a_{2}, \ldots, a_{n} \in \mathbb{F}$ there exists unique function $f \in V$ such that $f\left(x_{j}\right)=a_{j}$. 
Hence an $n$-dimensional space $V \subset \mathbb{F}[x]$ is Tchebycheff if and only if for any distinct set of points $\Delta:=\left\{x_{1}, x_{2}, \ldots, x_{n}\right\} \subset \mathbb{F}$, the space $V$ is complemented to an ideal

$$
J(\Delta):=\{f \in \mathbb{F}[x]: f(x)=0 \text { for all } x \in \Delta\}
$$

That is

$$
\mathbb{F}[x]=V \oplus J(\Delta)
$$

for any $\Delta \subset \mathbb{F}$ with cardinality $\# \Delta=n$.

An $n$-dimensional subspace $V \subset \mathbb{F}[x]$ is called an Extended Tchebycheff space (cf. [14], [15]) if every non-zero $f \in V$ has at most $n$ zeroes, counting multiplicity.

Equivalently (cf. [13]) an $n$-dimensional space $V \subset \mathbb{F}[x]$ is Extended Tchebycheff if and only if it is Hermite interpolating:

For any distinct set of $m \leq n$ points $\Delta:=\left\{x_{1}, x_{2}, \ldots, x_{m}\right\} \subset \mathbb{F}$, any set of integers $\mathfrak{N}(m, n)=\left\{n_{1}, \ldots n_{m}\right\}$ with $\sum_{j=1}^{m}\left(n_{j}-1\right)=n$ and any set of $n$ values $\left\{a_{1}^{\left(k_{1}\right)}, a_{2}^{\left(k_{2}\right)}, \ldots, a_{m}^{\left(k_{m}\right)}: k_{j}=0, \ldots, n_{j}-1\right\} \subset \mathbb{F}$ there exists a unique function $f \in V$ such that

$$
f^{\left(k_{j}\right)}\left(x_{j}\right)=a_{j}^{\left(k_{j}\right)}
$$

where $f^{(k)}$ denotes the $k$-th derivative of $f$.

Hence an $n$-dimensional space $V \subset \mathbb{F}[x]$ is Extended Tchebycheff if and only if for any distinct set of $m \leq n$ points $\Delta:=\left\{x_{1}, x_{2}, \ldots, x_{m}\right\} \subset \mathbb{F}$ any set of integers $\mathfrak{N}(m, n)=\left\{n_{1}, \ldots n_{m}\right\}$ with $\sum_{j=1}^{m}\left(n_{j}-1\right)=n$, the space $V$ is complemented to an ideal

$$
J(\Delta, \mathfrak{N}):=\left\{f \in \mathbb{F}[x]: f^{\left(k_{j}\right)}\left(x_{j}\right)=0 ; j=1, \ldots, m ; k_{j}=0, \ldots, n_{j}\right\} .
$$

That is

$$
\mathbb{F}[x]=V \oplus J(\Delta, \mathfrak{N}) .
$$

The reformulations (1.1) and (1.2) of the definitions of Tchebycheff and Extended Tchebycheff spaces motivates the definition of Ideal Complements as the $n$-dimensional spaces $V \subset \mathbb{F}[x]$ which are complemented to every ideal $J \subset \mathbb{F}[x]$ of codimension $n$.

An ideal $J \subset \mathbb{F}[x]$ is a subspace of $\mathbb{F}[x]$ such that

$$
f \in \mathbb{F}[x], g \in J \Longrightarrow f g \in J .
$$

Let $\mathfrak{J}$ be the set of all ideals in $\mathbb{F}[x]$ and let $\mathfrak{J}_{n} \subset \mathfrak{J}$ be the set of all ideals of codimension $n$.

Definition 1.1 An n-dimensional space $V \subset \mathbb{F}[x]$ is called an ideal complement if

$$
\mathbb{F}[x]=V \oplus J
$$

for every ideal $J \in \mathfrak{J}_{n}$ 
Clearly every ideal complement is an Extended Tchebycheff space and every Extended Tchebycheff space is Tchebycheff.

Since $\mathbb{F}[x]$ is a principle ideal domain (cf. [1]),

$$
J \in \mathfrak{J} \text { iff } J=p \mathbb{F}[x]
$$

for some polynomial $p \in \mathbb{F}[x]$.

Theorem 1.2 We have

1) If $p$ is a polynomial of degree $n$. Then $p \mathbb{F}[x] \in \mathfrak{J}_{n}$ and

$$
\mathbb{F}[x]=\mathbb{F}_{<n}[x] \oplus(p \mathbb{F}[x]) .
$$

In particular $\mathbb{F}_{<n}[x]$ is an ideal complement.

2) An ideal $J \in \mathfrak{J}_{n}$ iff $J=p \mathbb{F}[x]$ for some $p \in \mathbb{F}[x]$ with $\operatorname{deg} p=n$.

In particular every ideal $J \in \mathfrak{J}$ is of finite codimension.

Proof. If $p$ is a polynomial of degree $n$, then every non-zero polynomial in $p \mathbb{F}[x]$ has degree at least $n$. Hence $\mathbb{F}_{<n}[x] \cap(p \mathbb{F}[x])=\{0\}$. On the other hand every $f \in \mathbb{F}[x]$ can be written as $f=p q+r$ with $\operatorname{deg} r<n$. That proves the first part of the theorem. It also shows that $p \mathbb{F}[x]$ is an ideal of codimension $n$. To verify the rest of 2 ), assume that $J \in \mathfrak{J}_{n}$. Then there exists a polynomial $q$ such that $J=q \mathbb{F}[x]$. If $\operatorname{deg} q \neq n$ then, by part 1$)$, codim $J \neq n$ which gives the contradiction.

The last theorem shows that $\mathbb{F}_{<n}[x]$ is an ideal complement. In particular $\mathbb{F}_{<n}[x]$ is an Extended Tchebycheff space. Of course this is nothing new, except that the division algorithm used in the proof of the Theorem did not employ any determinants or complicated construction of basic polynomials!

In the next section we show $\mathbb{C}_{<n}[x]$ is the unique $n$-dimensional Tchebycheff subspace in $\mathbb{C}[x]$ and therefore it is the unique ideal complement in $\mathbb{C}[x]$. In particular, Tchebycheff spaces, Extended Tchebycheff spaces and ideal complements coincide.

In section 3 , we show that for $n>1$ the space $\mathbb{R}_{<n}[x]$ is the unique ideal complement in $\mathbb{R}[x]$ but not a unique Tchebycheff or Extended Tchebycheff subspace.

\subsection{Complex Case}

We start with the quick corollary of Theorem 1.2:

Theorem 1.3 The space $V=\mathbb{C}_{<n}[x]$ is the unique subspace of $\mathbb{C}[x]$ which complements every $J \in \mathfrak{J}_{n}$.

Proof. Let $V \neq \mathbb{C}_{<n}[x]$ be an $n$-dimensional subspace of $\mathbb{C}[x]$. Then $V$ contains a polynomial $q$ of degree $\geq n$. Hence $q=p f$ with $\operatorname{deg} p=n$. Let $J=p \mathbb{C}[x]$. By proposition $J \in \mathfrak{J}_{n}$ and $q \in V \cap J$. Thus $V$ is not complemented to $J$.

For the Tchebycheff spaces we have: 
Theorem 1.4 The space $V=\mathbb{C}_{<n}[x]$ is the unique $n$-dimensional Tchebycheff subspace of $\mathbb{C}[x]$.

Proof. Suppose that $V \neq \mathbb{C}_{\leq n}[x]$ is an $n$-dimensional Tchebycheff subspace of $\mathbb{C}[x]$. Then $V$ contains a polynomial $f$ with $\operatorname{deg} f \geq n$. Since $V$ is Tchebycheff, hence $f$ has at most $n-1$ zeroes: $\xi_{1}, \ldots, \xi_{k}$ with $k<n$. Once again, since $V$ is Tchebycheff, there exists a polynomial $g \in V$, such that $g\left(\xi_{j}\right)=1$ for all $j=1, \ldots, k$. Hence $f$ and $g$ are relative primes and $\left(\frac{f}{g}\right)^{\prime}$ is different from 0 . For every $c \in \mathbb{C}$, consider a new polynomial $q(c, x)=f(x)-c g(x)$. We now claim that for all, but a finite many values of $c \in \mathbb{C}$, the polynomial $q(c, x)$ has only simple zeroes. Indeed let $\zeta_{1}, \ldots, \zeta_{N}$ be all the zeroes of the polynomial $f g^{\prime}-g f^{\prime}$ and assume that

$$
c \neq \frac{f\left(\zeta_{j}\right)}{g\left(\zeta_{j}\right)}
$$

for those $\zeta_{j}$, for which $g\left(\zeta_{j}\right) \neq 0$. Then if $x_{0}$ is a multiple root of $f(x)-c g(x)$, we have

$$
\left\{\begin{array}{c}
f\left(x_{0}\right)-c g\left(x_{0}\right)=0 \\
f^{\prime}\left(x_{0}\right)-c g^{\prime}\left(x_{0}\right)=0
\end{array} .\right.
$$

Since $f$ and $g$ are relative primes, hence $g\left(x_{0}\right) \neq 0$. From the first of the equations above, we obtain $c=\frac{f\left(x_{0}\right)}{g\left(x_{0}\right)}$ and substituting it into the second equation, we have $f\left(x_{0}\right) g^{\prime}\left(x_{0}\right)-g\left(x_{0}\right) f^{\prime}\left(x_{0}\right)=0$, which contradicts (1.5).

Corollary 1.5 The space $V=\mathbb{C}_{<n}[x]$ is the unique $n$-dimensional Extended Tchebycheff subspace of $\mathbb{C}[x]$.

\subsection{Real Case}

Real ideal complements have almost the same description as complex ideal complements.

Theorem 1.6 Let $V$ be an $n$-dimensional ideal complement. If $n>1$ then $V=\mathbb{R}_{<n}[x]$. If $n=1$, then $V$ is the set of constant multiples of any strictly positive polynomial; $V=\operatorname{span}\{p\}, p \in \mathbb{R}[x]$ and $p>0$.

Proof. Let $n>1$ and let $\xi_{1}, \ldots, \xi_{n}$ be distinct points in $\mathbb{R}$. Since $V$ is an ideal complement, in particular it is a Tchebycheff subspace. Hence there are $n$ polynomials $p_{1}, \ldots, p_{n} \in V$ such that $p_{k}\left(\xi_{j}\right)=\delta_{j, k}$. These polynomials are linearly independent and thus span the space $V$. If $\max \{\operatorname{deg} p: p \in V\} \geq n$ then at least one of the polynomials, say $p_{1}$ has degree greater then $n-1$. Since $p_{1}$ has a linear factor, it follows that $p_{1}$ has a factor of degree $n$ and hence $V$ is not an ideal complement. The case $n=1$ is trivial.

Corollary 1.7 For $n=1$ an $n$-dimensional space $V$ is Tchebycheff if and only if it is an ideal complement. For $n>1$ there exists an $n$-dimensional Tchebycheff subspace of $\mathbb{R}[x]$ which is not an ideal complement. 
Proof. Any subspace $V \subset \mathbb{R}[x]$ which is of the form

$$
V=r(x) \mathbb{R}_{<n}[s(x)]:=\operatorname{span}\left\{r(x), r(x) s(x), \ldots, r(x) s^{n-1}(x)\right\}
$$

where $r(x)$ is a strictly positive polynomial in $\mathbb{R}[x]$ and $s$ is an injective polynomial mapping from $\mathbb{R} \rightarrow \mathbb{R}$ is clearly a Tchebycheff space. Yet if $\operatorname{deg} r>0$ then, as follows from the previous Theorem, it is not an ideal complement.

This argument leads to the a reasonable possibility that a subspace $V \subset$ $\mathbb{R}[x]$ is Tchebycheff if and only if $V=r(x) \mathbb{R}_{<n}[s(x)]$ for some strictly positive polynomial $r(x)$ and an injective polynomial mapping $s$ from $\mathbb{R} \rightarrow \mathbb{R}$.

This is clearly true for $n=1$.

Unfortunately this is not so for $n>1$. Indeed here is a counterexample:

Let

$$
V:=\operatorname{span}\left\{1+x^{2}, x^{3}\right\} .
$$

We have

$$
\begin{aligned}
\operatorname{det}\left[\begin{array}{ll}
1+x^{2} & x^{3} \\
1+a^{2} & a^{3}
\end{array}\right] & =-(x-a)\left(x^{2} a^{2}+x^{2}+x a+a^{2}\right) \\
& =-(x-a)\left(x^{2} a^{2}+\frac{1}{2}\left(x^{2}+a^{2}+(x+a)^{2}\right)\right)
\end{aligned}
$$

which is equal to zero if and only if $x=a$. Hence $V$ is a Tchebycheff space that is not of the form (1.6).

Furthermore

$$
\operatorname{det}\left[\begin{array}{cc}
1+x^{2} & x^{3} \\
2 x & 3 x^{2}
\end{array}\right]=3 x^{2}+x^{4}=0 \text { if } x=0 .
$$

Hence the Tchebycheff space $V$ defined by (1.7) is not an Extended Tchebycheff space.

On the other hand the space

$$
V=\operatorname{span}\left\{x^{2}+1, x^{3}+2 x\right\}
$$

is an Extended Tchebycheff space. Indeed

$$
\operatorname{det}\left[\begin{array}{cc}
x^{2}+1 & x^{3}+2 x \\
2 x & 3 x^{2}+2
\end{array}\right]=x^{4}+x^{2}+1>0 .
$$

And if $x \neq a$ then

$$
\begin{aligned}
\operatorname{det}\left[\begin{array}{ll}
x^{2}+1 & x^{3}+2 x \\
a^{2}+1 & a^{3}+2 a
\end{array}\right] & =-(x-a)\left(x^{2} a^{2}+x^{2}-x a+a^{2}+2\right) \\
& =-(x-a)\left(\frac{1}{4}(2 x-a)^{2}+x^{2} a^{2}+\frac{3}{4} a^{2}+2\right) \neq 0
\end{aligned}
$$


Conclusion 1.8 In $\mathbb{R}[x]$ there are Tchebycheff spaces which are not Extended Tchebycheff spaces and there are Extended Tchebycheff spaces which are not of the form (1.6).

Problem 1.9 What is the general form of Tchebycheff spaces in $\mathbb{R}[x]$ ? What is the general form of Extended Tchebycheff spaces in $\mathbb{R}[x]$ ?

Now suppose that $1 \in V$ and $V$ is a Tchebycheff space. Does that imply that

$$
V=\mathbb{R}_{<n}[s(x)] ?
$$

For $n=2$ it is so. Indeed if $V=\operatorname{span}\{1, s(x)\}$ then $s$ is strictly monotone and hence an injection.

Our next example shows that (1.8) fails for $n=3$ :

Let $V=\operatorname{span}\left\{1, x, x^{4}\right\}$. Then

$$
\begin{aligned}
\operatorname{det}\left[\begin{array}{lll}
1 & x & x^{4} \\
1 & a & a^{4} \\
1 & b & b^{4}
\end{array}\right] & =a b^{4}-a^{4} b-x b^{4}+x^{4} b+x a^{4}-x^{4} a \\
& =-\frac{1}{2}(-b+a)(x-b)(x-a)\left(\left(x^{2}+a^{2}+b^{2}\right)+(x+a+b)^{2}\right)
\end{aligned}
$$

Hence $\operatorname{span}\left\{1, x, x^{4}\right\}$ is a Tchebycheff space that is not an ideal complement.

\section{Interpolation Systems in Several Variables.}

Let $\mathbb{F}\left[x_{1}, \ldots, x_{d}\right]=\mathbb{F}[\mathbf{x}]$ be the ring of polynomials of $d$ variables and let $\mathbb{F}_{\leq m}[\mathbf{x}]$ be the space of polynomials of degree at most $m$. For an ideal $J \subset \mathbb{F}[\mathbf{x}]$ we define

$$
Z(J):=\left\{\mathbf{x} \in \mathbb{F}^{d}: f(x)=0 \text { for all } f \in J\right\} .
$$

If an ideal $J \subset \mathbb{F}$ is generated by polynomials $f_{1}, f_{2} \ldots, f_{n}$, we use the standard notation:

$$
J=<f_{1}, f_{2} \ldots, f_{n}>=<f_{j}: j=1, \ldots, n>.
$$

Let, once again, $\mathfrak{J}_{n}$ denotes the family of ideals of codimension $n$.

For ideal $J \in \mathfrak{J}_{n}$, the set $Z(J)$ is finite and moreover

$$
\# Z(J) \leq n
$$

An ideal $J \subset \mathbb{F}$ is called radical if $f^{m} \in J$ implies $f \in J$. It is well known (cf. [9]) that an ideal $J \in \mathfrak{J}_{n}$ is radical if and only if $\# Z(J)=n$.

In several variables there are no Tchebycheff subspaces and therefore there are no ideal complements. For the real field this follows from extremely cute "Mairhuber argument (cf [15])":

Let $V=\operatorname{span}\left[f_{1}, f_{2}, \ldots, f_{n}\right]$. And let $\Delta=\left\{\mathbf{x}_{1}, \mathbf{x}_{2}, \mathbf{x}_{3}, \ldots, \mathbf{x}_{n}\right\}$ be distinct points in $\mathbb{R}^{d}$ with $d \geq 2$. Position two points $\mathbf{x}_{1}, \mathbf{x}_{2}$ on diametrically opposite 
ends of the unit circle and points $\mathbf{x}_{3}, \ldots, \mathbf{x}_{n}$ outside the circle. If the space $V$ is Tchebycheff, that implies that the determinant

$$
D(\Delta)=\operatorname{det}\left[f_{k}\left(\mathbf{x}_{j}\right)\right] \neq 0
$$

for any $\Delta$. As we rotate the diameter, the points $\mathbf{x}_{1}$ and $\mathbf{x}_{2}$ switch positions and hence $D(\Delta)$ changes sign. By the intermediate value theorem, there exists a pair $\mathbf{x}_{1}, \mathbf{x}_{2}$ such that $D(\Delta)=0$; hence $V$ is not interpolating at these points.

In the absence of an intermediate value theorem, the complex case utilizes different tools. Since this article deals with polynomials, we present an argument based on the attributes from Algebraic Geometry:

Let

$$
Z:=\left\{\left(\mathbf{x}_{1}, \mathbf{x}_{2}, \mathbf{x}_{3}, \ldots, \mathbf{x}_{n}\right) \in \mathbb{C}^{n \cdot d}: D(\Delta)=\operatorname{det}\left[f_{k}\left(\mathbf{x}_{j}\right)\right]=0\right\}
$$

Let

$$
U_{j, k}:=\left\{\left(\mathbf{x}_{1}, \mathbf{x}_{2}, \mathbf{x}_{3}, \ldots, \mathbf{x}_{n}\right) \in \mathbb{C}^{n \cdot d}: \mathbf{x}_{j}=\mathbf{x}_{k}\right\} \text { and } U:=\bigcup_{j \neq k}^{\cup} U_{j, k}
$$

Since $Z$ is the set of solutions of one equation $D(\Delta)=0$ in $\mathbb{C}^{n d}$, hence $Z$ is an algebraic variety of codimension one, thus $\operatorname{dim} Z=n d-1$. Each $U_{j, k}$ is the zero locus of $d$ equations: $\mathbf{x}_{j}=\mathbf{x}_{k}$, and hence it is a variety of codimension $d$. We conclude that for $d>1$ :

$$
\operatorname{dim} U=\max \operatorname{dim} U_{j, k}=n d-d<n d-1=\operatorname{dim} Z .
$$

Hence there exists an $n$-tuple $\left(\mathbf{x}_{1}, \mathbf{x}_{2}, \mathbf{x}_{3}, \ldots, \mathbf{x}_{n}\right) \in Z$ which is not in $U$. Thus the equation

$$
D(\Delta)=\operatorname{det}\left[f_{k}\left(\mathbf{x}_{j}\right)\right]=0
$$

has a solution for some set $\Delta$ of distinct points in $\mathbb{C}^{d}$, which implies that $V$ is not a Tchebycheff space. In the absence of Tchebycheff Spaces in several variables, we have to give something up. We propose two possible analogues of Tchebycheff spaces.

\subsection{Interpolating Spaces}

Definition 2.1 A subspace $V \subset \mathbb{F}[\mathbf{x}]$ is called $k$-interpolating if for every $k$ distinct points $\mathbf{x}_{1}, \ldots, \mathbf{x}_{k}$ in $\mathbb{F}^{d}$ and for every distinct values $\alpha_{1}, \ldots, \alpha_{k}$ there exists $f \in V$ such that

$$
f\left(\mathbf{x}_{j}\right)=\alpha_{j} ; j=1, \ldots, k .
$$

Clearly if $V$ is $k$-interpolating then $\operatorname{dim} V \geq k$. If $\operatorname{dim} V=k$ then $V$ is $k$ interpolating if and only if $V$ is Tchebycheff. As we mentioned earlier for $d>1$ Tchebycheff spaces do not exist. The $k$-interpolating spaces do exist. That means that we give up uniqueness of the interpolating function $f \in V$ but still insist on the existence of one. However we do not want to abandon uniqueness all together. One way of doing so is to ask for a $k$-interpolating space of minimal dimension. Therefore the problem in several variables can be reformulated as follows: 
Problem 2.2 What is the minimal dimension of $k$-interpolating spaces in $\mathbb{F}[\mathbf{x}]$ ? What are the $k$-interpolating subspaces of $\mathbb{F}[\mathbf{x}]$ of minimal dimension.

Just as in the last section, we can observe that a space $V$ is $k$-interpolating if and only if for every radical ideal $J \in \mathbb{F}[\mathbf{x}]$ of codimension $k$ there exists a subspace $E \subset V$ such that

$$
E \oplus J=\mathbb{F}[\mathbf{x}] .
$$

Hence it seems natural to extend this definition to all ideals.

Definition 2.3 A space $V \subset \mathbb{F}[\mathbf{x}]$ is called a $k$-ideal complement if for every ideal $J$ of codimension $k$ there exists a subspace $E \subset V$ such that

$$
E \oplus J=\mathbb{F}[\mathbf{x}] .
$$

Once again we have the problem:

Problem 2.4 What is the minimal dimension of a $k$-ideal complement? What are the $k$-ideal complements of minimal dimension? Are the minimal k-ideal complements unique? Do the minimal k-ideal complements coincide with the minimal $k$-interpolating spaces.

There are some results ( cf. [8], [22], [23], [24], [25]) concerning the minimal dimension of $k$-interpolating subspaces in $\mathbb{R}[\mathbf{x}]$. The most stunning of these is due to F. Cohen and D. Handel [8] (cf. also [24]):

Theorem 2.5 Let $a(k)$ be the minimal dimension of $k$-interpolating subspaces in $\mathbb{R}[x, y]$. Then

$$
2 k-\eta(k) \leq a(k) \leq 2 k-1,
$$

where $\eta(k)$ is the number of 1 's in the binary representation of the integer $n$.

In fact for $k=3$ the value $a(3)=4$ as the "unnatural" appearance of $\eta(k)$ in the lower bound would predict $(\operatorname{cf}[22])$. A minimal 3-interpolating subspace is spanned by polynomials $\left\{1, x, y, x^{2}+y^{2}\right\}$. For $k=4$ the lower and the upper bounds coincide. Hence $a(4)=7$. A minimal 4 -interpolating subspace is spanned by polynomials $\left\{1, x, y, x^{2}-y^{2}, x y, x^{3}-3 x y^{2}, y^{3}-3 x^{2} y\right\}$.

To the best of my knowledge, the exact value for $a(5)$ is not known. The span of the first $2 k-1$ harmonic polynomials always forms a $k$-interpolating subspace in $\mathbb{R}[x, y]$. For $d>2$, the only reasonable bound known to me (cf. $[22],[23])$ is

$$
\frac{1}{2}(d+1) k \leq a(k) \leq d(k+1) .
$$

As far as I know there are no results on minimal $k$-dimensional interpolating subspaces in the complex case. The ideal complements of minimal dimension have not been studied in either field.

It follows from the Theorem 2.9 mentioned in the next section, that the space $\mathbb{F}_{<k}[\mathbf{x}]$ is a $k$-ideal complement.

Now the standard transversality argument (cf. [21], [23]) gives us a better estimate: 
Theorem 2.6 There exists a $k$-ideal complement $V \subset \mathbb{F}_{<k}[\mathbf{x}]$ with $\operatorname{dim} V \leq$ $(d+1) k$.

\subsection{Interpolating Families in Several Variables.}

As we mentioned in the previous section, the four-dimensional space spanned by polynomials $\left\{1, x, y, x^{2}+y^{2}\right\}$ is 3 -interpolating in $\mathbb{R}[x, y]$. Indeed if we have three points $u_{1}, u_{2}, u_{3} \in \mathbb{R}^{2}$ that do not lie on the same line, then the threedimensional space spanned by $\{1, x, y\}$ interpolates at those points. On the other hand if three distinct points $u_{1}, u_{2}, u_{3} \in \mathbb{R}^{2}$ do lie on the same line, then either the space spanned by $\left\{1, x, x^{2}+y^{2}\right\}$ or by $\left\{1, y, x^{2}+y^{2}\right\}$ interpolate at those points. In other words in order to accomplish the interpolation at arbitrary three points, we do not need all (infinitely many) three-dimensional subspaces of $\operatorname{span}\left\{1, x, y, x^{2}+y^{2}\right\}$. It is sufficient to consider three of them:

$$
\operatorname{span}\{1, x, y\}, \operatorname{span}\left\{1, x, x^{2}+y^{2}\right\} \text { and } \operatorname{span}\left\{1, y, x^{2}+y^{2}\right\} .
$$

This consideration prompts the following definition:

Definition 2.7 A family $\mathcal{F}$ of $k$-dimensional subspaces of $\mathbb{F}[\mathbf{x}]$ is called a family of $k$-ideal complements, if for every ideal $J \subset \mathbb{F}[\mathbf{x}]$ of codimension $k$ there exists a subspace $E \in \mathcal{F}$ such that

$$
E \oplus J=\mathbb{F}[\mathbf{x}] .
$$

A family $\mathcal{F}$ of $k$-dimensional subspaces of $\mathbb{F}[\mathbf{x}]$ is called a $k$-interpolating family if for every radical ideal $J \subset \mathbb{F}[\mathbf{x}]$ of codimension $k$ there exists a subspace $E \in \mathcal{F}$ such that

$$
E \oplus J=\mathbb{F}[\mathbf{x}] .
$$

With these definitions come apparent open questions:

Problem 2.8 What is the minimal number of subspaces in a family of $k$-ideal complements? What is the minimal number of subspaces in a $k$-interpolating family?

A subspace $V \subset \mathbb{F}\left[x_{1}, \ldots, x_{d}\right]=\mathbb{F}[\mathbf{x}]$ is called $D$-invariant if

$$
f \in V \Longrightarrow \frac{\partial}{\partial x_{j}} f \in V, \forall j=1, \ldots, d .
$$

The next theorem was first proved in [11]. The introduction of Groebner bases, made it a simple theorem ( $\mathrm{cf}[3])$ :

Theorem 2.9 For every $J \in \mathfrak{J}_{n}$ there exists a $D$-invariant subspace $V \subset \mathbb{F}[\mathbf{x}]$ spanned by monomials, such that

$$
V \oplus J=\mathbb{F}[\mathbf{x}] .
$$


A moment of reflection on $D$-invariance and monomial nature of this space leads to the conclusion that every such space is a subspace of $\mathbb{F}_{<n}[\mathbf{x}]$ and since there are only finitely many monomials in $\mathbb{F}_{<n}[\mathbf{x}]$, hence there are only finitely many such spaces.

Corollary 2.10 There exist a finite $k$-ideal family.

It is convenient to use Young tables to visualize such subspaces. For instance for $n=4$ the five subspaces in question are given by tables (staircases):

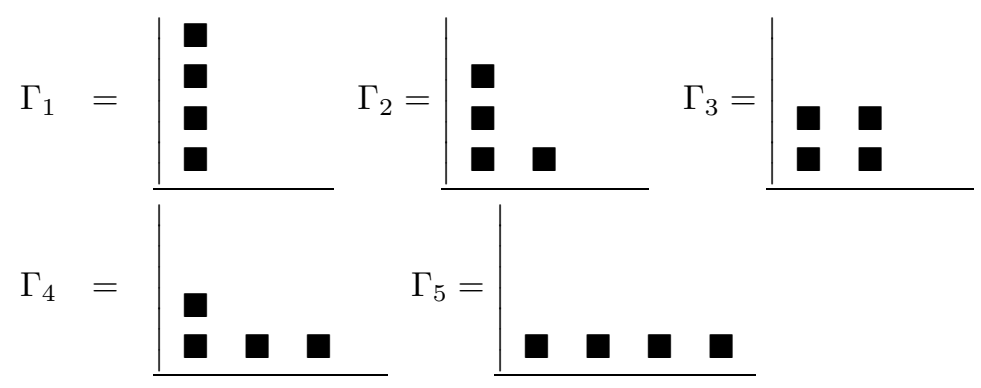

These five tables represent all possible $D$-invariant complements to ideals in $\mathfrak{J}_{4}$. Thinking of the vertical axes as the number of monomials in $y$, we can write all five gammas as

$$
\begin{aligned}
& \Gamma_{1}=\left[1, y, y^{2}, y^{3}\right], \Gamma_{2}=\left[1, y, y^{2}, x\right], \Gamma_{3}=[1, y, x, x y] \\
& \Gamma_{4}=\left[1, y, x, x^{2}\right], \Gamma_{5}=\left[1, x, x^{2}, x^{3}\right] .
\end{aligned}
$$

Now the spaces $G_{j}:=\operatorname{span} \Gamma_{j}$ represent the five complements.

Clearly no four of those subspaces can serve the same purpose, for an ideal generated by, say $\left\langle x^{4}, y\right\rangle \in \mathfrak{J}_{4}$ is not complemented to the first four subspaces. It is also easy to see that the minimal 2-interpolating and 2-ideal family is

$$
\mathcal{F}=\{\operatorname{span}\{1, x\}, \operatorname{span}\{1, y\}\},
$$

since this family is 2-ideal, by the last theorem, and no one two-dimensional subspace is 2-interpolating, by the results of the previous section.

Theorem 2.11 The minimal number of subspaces in a family of 3-ideal complements in $\mathbb{C}[x, y]$ is 3 .

Proof. It follows from the Theorem 2.9 , that the family $\mathcal{F}$ consisting of three spaces:

$$
\operatorname{span}\left\{1, x, x^{2}\right\}, \operatorname{span}\{1, x, y\} \text { and } \operatorname{span}\left\{1, y, y^{2}\right\}
$$

is a $k$-ideal family. Thus it remains to proof that no two three-dimensional spaces form a family of $k$-ideal complements. Let the subspaces $V_{1}$ and $V_{2}$ form such a family. Consider several cases: 
Case 1: There exists a non-constant polynomial $p \in \mathbb{C}[x, y]$ and polynomials $f_{k} \in V_{k}$ such that $f_{k}=h_{k} \cdot p$. Then consider the set

$$
Z:=\left\{(x, y) \in \mathbb{C}^{2}: p(x, y)=0\right\} .
$$

This is an infinite set and hence contains three distinct points

$$
\left(x_{1}, y_{1}\right),\left(x_{2}, y_{2}\right),\left(x_{3}, y_{3}\right) \in Z \text {. }
$$

Next consider the ideal

$$
J:=\left\{f \in \mathbb{C}[x, y]: f\left(x_{j}, y_{j}\right)=0, j=1,2,3\right\} .
$$

Clearly, $J$ is a radical ideal in $\mathfrak{J}_{3}$. Since for $k=1,2$ we have $f_{k}\left(x_{j}, y_{j}\right)=0$ and since $J$ is a radical, this implies that $f_{k} \in J$ and hence $f_{k} \in V_{k} \cap J \neq\{0\}$. In particular, neither $V_{1}$ nor $V_{2}$ complement $J$ and $\left\{V_{1}, V_{2}\right\}$ is not a $k$-ideal family.

Case 2.: Suppose that

$$
N:=\max \operatorname{deg}\left\{f: f \in V_{1}\right\} \cdot \max \operatorname{deg}\left\{f: f \in V_{2}\right\} \geq 3 .
$$

Let $f_{k} \in V_{k}$ be any polynomials, such that

$$
\operatorname{deg} f_{k}=\max \operatorname{deg}\left\{f: f \in V_{k}\right\}, k=1,2,
$$

then (by Case 1) they do not contain a common non-zero factor. By Bezout's Theorem (cf. [9]), there exist $N \geq 3$ solutions (counting multiplicity) to the set of equations

$$
f_{k}(x, y)=0, k=1,2 .
$$

Hence, once again, there exist an ideal $J \in \mathfrak{J}_{3}$ such that $f_{k} \in V_{k} \cap J \neq\{0\}$.

Case 3. The last remaining case is the case, when $V_{1}$ consists of polynomials of degree one and $V_{2}$ consists of polynomials of degree two and does not contain any non-constant linear polynomial. Since $\operatorname{dim} V_{k}=3$, hence $V_{1}=\operatorname{span}\{1, x, y\}$ and $V_{2}$ is spanned by three quadratic polynomials and does not contain a nonconstant linear function. We claim that at least one polynomial in $V_{2}$ has a linear factor, thus reducing this case to Case 1 . Indeed, suppose that $V_{2}$ is spanned by quadratic polynomials $\left\{f_{j}(x, y), j=1,2,3\right\}$. Consider the polynomial

$$
p(x):=\sum_{j=1}^{3} a_{j} f_{j}(x, A x+B) \in V_{2} .
$$

This is a quadratic polynomial with three coefficients that depend on five parameters: $A, B, a_{j}$. Setting these coefficients to zero, we obtain three equations in five unknowns, which clearly have a solution in $\mathbb{C}$. Thus the polynomial

$$
\sum_{j=1}^{3} a_{j} f_{j}(x y)
$$

has a linear factor: $y-A x-B$. 


\section{References}

[1] Atiyah, M. F. and MacDonald, I. G. Introduction to Commutative Algebra. Reading, MA: Addison-Wesley, pp. 9-10, 1969

[2] Birkhoff, G. The Algebra of Multivariate Interpolation, in "Constructive approaches to mathematical models." (C.V. Coffman and G.J.Fix Eds.), pp345-363, Academic Press, New-York, 1979

[3] de Boor, C. , Ideal Interpolation., in Approximation Theory XI: Gatlinburg 2004, C.K. Chui, M. Neamtu, and L.L. Schumaker (eds.). Nasboro Press, 2005, pp 59-91.

[4] de Bruin, Marcel G.; Sharma, A. Birkhoff interpolation on nonuniformly distributed roots of unity. II. Proceedings of the Fifth International Symposium on Orthogonal Polynomials, Special Functions and their Applications (Patras, 1999). J. Comput. Appl. Math. 133 (2001), no. 1-2, 295-303.

[5] de Bruin, Marcel G.; Sharma, A. Birkhoff interpolation on non-uniformly distributed roots of unity. Mathematical journey through analysis, matrix theory and scientific computation (Kent, OH, 1999). Numer. Algorithms 25 (2000), no. 1-4, 123-138.

[6] de Bruin, M. G.; Sharma, A.; Szabados, J. Birkhoff type interpolation on perturbed roots of unity. Approximation theory, 167-179, Monogr. Textbooks Pure Appl. Math., 212, Dekker, New York, 1998.

[7] Bokhari, M. A.; Dikshit, H. P.; Sharma, A. Birkhoff interpolation on some perturbed roots of unity: revisited. Mathematical journey through analysis, matrix theory and scientific computation (Kent, OH, 1999). Numer. Algorithms 25 (2000), no. 1-4, 47-62.

[8] Cohen, F. R.; D. Handel, k-regular embeddings of the plane, Proc. AMS, 72 (1978), 202-204.

[9] Cox, D., J. Little and D. O'Shea, Ideals,Varieties, and Algorithms, Springer-Verlag, New-York-Berlin-Heidelberg, 1997.

[10] Fabrykowski, J.; Sharma, A.; Zassenhaus, H. Some Birkhoff interpolation problems on the roots of unity. Linear Algebra Appl. 65 (1985), 1-23.

[11] Gordan, M. Les Invariants des Formes Binaries, J. Math. Pures et Appli. (Liuville's J.) 6 (1900) pp.141-156.

[12] Jia, Rong Qing; Sharma, A. Solvability of some multivariate interpolation problems. J. Reine Angew. Math. 421 (1991), 73-81.

[13] Karlin, S. ;W. J. Studden, Tchebycheff systems: with applications in analysis and statistics, Interscience, New York, 1966. 
[14] Krein, M.G.; A.A. Nudelman: The Markov Moment Problem and Extremal Problems, Amer. Math. Soc. Transl. Math. Monographs, Providence, RI, 1977.

[15] Mairhuber, J.C. On Haar's Theorem Concerning Chebyshev Approximation Problems Having Unique Solution, Proc. AMS. 7(1956) 609-615.

[16] Sharma, A.; Varga, R. S. On a 2-periodic lacunary trigonometric interpolation problem. Approximation theory VI, Vol. II (College Station, TX, 1989), 585-588, Academic Press, Boston, MA, 1989.

[17] Sharma, A.; Varga, R. S. On a particular 2-periodic lacunary trigonometric interpolation problem on equidistant nodes. Results Math. 16 (1989), no. 3-4, 383-404.

[18] Sharma, A.; Ziegler, Z. Hermite interpolation on some perturbed roots of unity. Analysis (Munich) 19 (1999), no. 1, 1-12.

[19] Sharma, A.; Szabados, J.; Underhill, B.; Varma, A. K. On some general lacunary interpolation problems. J. Approx. Theory 87 (1996), no. 2, 194219.

[20] Shekhtman, B., Case Study in Hermite Interpolation, Journal of Approximation Theory (submitted)

[21] Shekhtman, B.,On Hermite Interpolation in $\mathbb{R}_{d}$, ETNA 18 (2004), pp. 6572 .

[22] Shekhtman, B.,Interpolating subspaces in $\mathbb{R}_{n}$, in Approximation Theory, Wavelets and Applications, S.P.Singh(ed), Kluwer, 1995, pp.465-471.

[23] Shekhtman, B., Interpolation by Polynomials in Several Variables, Approximation Theory X (2001), C.K. Chui, L.L. Schumaker and J. Stoekler (eds.), Vanderbilt Univ. Press, (2002), pp367-372.

[24] Vasiliev, V.A., On Function Spaces that are Interpolating at every k nodes, Funct. Anal. Appl. (1992), 72-74.

[25] Wulbert, D., Interpolation at a Few Points, J. Approx. Theory, 96(1999), no1, 139-148. 